\title{
Reactions of Fungi to Exposure to 10 Atmospheres Pressure of Oxygen
}

\author{
By SHEILA M. ROBB \\ Department of Botany, University of Exeter
}

(Received 10 November 1965)

\begin{abstract}
SUMMARY
Of 103 species of fungi exposed to 10 atmospheres (atm.) pressure of oxygen at $25^{\circ}$ for 7 days 52 resumed growth after treatment. Of these, 22 recovered from a similar treatment for 14 days. On resumption of growth, growth rates were the same as those of untreated colonies, after a lag period between decompression and recovery. The lag period varied with species, length of exposure, inoculum source, and in some cases between replicates of a species. More detailed investigation of the reaction of Fusarium solani, Rhizopus arrhizus, Mucor racemosus and $M$. plumbeus showed that generally the lag periods increased with increasing exposure times, that their extinction points (i.e. the exposure which killed all replicates) varied, and that at exposures approaching the extinction point there was selective survival either of spores or of strains in the latter three fungi.
\end{abstract}

\section{INTRODUCTION}

The effects of high oxygen pressures on living organisms have been under investigation for many years. Studies have extended over a wide range of test organisms, including man (Bean, 1945). Deep-sea fish are known to live at a depth of $1365 \mathrm{~m}$. where the partial pressure of oxygen is equivalent to about $115 \mathrm{~atm}$. of pure oxygen. Frogs and an alligator have survived, apparently with no ill effects, after exposure to $3.5 \mathrm{~atm}$. of oxygen for $65 \mathrm{hr}$ and $4.3 \mathrm{~atm}$. of oxygen for $75 \mathrm{~min}$., respectively. A guinea pig and a dog subjected to the latter treatment did not survive, and this and other experiments suggested that cold-blooded animals are more tolerant of high oxygen pressures than are warm-blooded. Cleveland (1925) succeeded in killing the protozoan parasites in insects, earthworms, frogs, goldfish and salamanders by subjecting them to oxygen pressures of up to $3 \cdot 5 \mathrm{~atm}$. for periods well below those which killed the hosts. In similar experiments to free rats from protozoa, he found that the host was killed before the parasite. These effects are of great significance in the medical field, where high oxygen pressures are cautiously administered to human beings, e.g. in the resuscitation of new-born babies (Hutchison, 1963) and in the alleviation or cure of anaerobic infection (Boerema, Brummelkamp \& Miejne, 1964). Little is known, however, about the direct effect of high oxygen pressures on micro-organisms. Bert (1878) reported the inhibitory or destructive action of hyperoxygenated air at $10 \mathrm{~atm}$. on putrefactive organisms in wine and meat, on moulds, yeasts and Bacillus anthracis, although hyperoxygenated air at $20 \mathrm{~atm}$. did not kill the glanders organism. Of 20 micro-organisms studied by Berghaus (see Bean, 1945) 15 remained viable after $24 \mathrm{hr}$ at $35 \mathrm{~atm}$. of oxygen. 
Thaysen (1934) recorded that the growth of 4 micro-organisms was retarded by exposure to $10 \mathrm{~atm}$. pressure, and McAllister, Stark, Norman \& Ross (1963) reported that exposure of eight bacteria and two fungi to $2 \mathrm{~atm}$. of oxygen for $42 \mathrm{hr}$ temporarily inhibited growth and permanently altered three of the bacteria (Pseudomonas pyocyanea (aeruginosa), Staphylococcus aureus and Escherichia coli). Caldwell (1963, 1964, 1965) described the effect of high oxygen pressures on a variety of organisms, including fungi, bacteria, mites and dormant plant seeds. Bacteria appeared to be remarkably resistant to up to $10 \mathrm{~atm}$. of oxygen for up to 14 days, and $E$. coli, $S$. citreus, $S$. albus and a chromobacterium species recovered from a 28-day exposure (Caldwell, 1965). Fungi subjected to $10 \mathrm{~atm}$. pressure of oxygen varied in the length of exposure which they could tolerate. Aspergillus niger, Sordaria fimicola and Penicillium cyclopium recovered from exposure for 18 days, 14 days and 10 days, respectively. Caldwell noted that, whereas bacteria recover almost immediately after treatment, with fungi there was a lag period between decompression and resumption of growth, and that the lag period was longer with longer exposure at any one pressure, or with the same time of exposure at higher pressures. To investigate various aspects of the tolerance of a wider range of fungi the present work was undertaken.

\section{METHODS}

The species of fungi used in this investigation numbered 103 and comprised 26 Phycomycetes, 16 Ascomycetes, 2 Basidiomycetes and 59 Fungi Imperfecti. Four replicates of each were grown on malt agar $(25 \mathrm{~g}$. malt extract and $15 \mathrm{~g}$. agar/l.) in Petri dishes. Two plates were retained as controls in an incubator at $25^{\circ}$ and the diameter of the colonies was measured as an indication of growth rate. Two plates, after growing for 2 days at $25^{\circ}$ to ensure establishment, were placed in a pressure vessel, flushed out with oxygen, and then kept at $10 \mathrm{~atm}$. of oxygen in a constant temperature room at $25^{\circ}$ for various periods. At the end of the experimental period, the pressure was released slowly, the treated plates removed to an incubator at $25^{\circ}$, observed for 14 days, and any increases in colony diameter measured.

The objection might be raised that inhibitory effects on growth in pressure vessels first flushed out with oxygen (thus removing air) might be the result of lack of $\mathrm{CO}_{2}$. This possibility was tested by introducing pure oxygen into unflushed pressure vessels, which therefore retained $\mathrm{CO}_{2}$ at atmospheric concentrations. Growth was inhibited in both flushed and unflushed vessels; therefore it was assumed that the absence of atmospheric concentrations of $\mathrm{CO}_{2}$ in flushed vessels was not the cause of inhibition, and that the latter was due to the high partial pressure of oxygen.

At first all cultures were subjected to pressure for 14 days. Subsequently, those fungi which did not recover after this treatment were subjected to pressure for 7 days. Finally, a more detailed study was made with Mucor plumbeus, M. racemosus, Fusarium solani and Rhizopus arrhizus to elucidate points which arose during the previous survey.

\section{RESULTS}

Twenty-two fungi recovered from exposure to $10 \mathrm{~atm}$. of oxygen for 14 days (Table 1). They were 6 Phycomycetes (Achlya bisexualis, Circinella mucoroides, Mucor pusillus, M. ramannianus, Syncephalastrum cinereum, Thamnidium elegans); 
2 Ascomycetes (Gymnoascus subumbrinus, Sordaria fimicola); and 14 Fungi Imperfecti (Aspergillus amstelodami, A. chevalieri, A. flavus, A. fumigatus, A. niger, $A$. quercinus, $A$. tamarii, $A$. terreus, Penicillium chrysogenum, $P$. cyclopium, $P$. cylindrosporium, $\boldsymbol{P}$. decumbens, $\boldsymbol{P}$. varioti, $\boldsymbol{P}$. velutinum).

Thirty fungi were resistant to exposure for 7 days, but not to 14 days, to $10 \mathrm{~atm}$. of oxygen. They were 7 Phycomycetes (Absidia glauca, Mucor plumbeus, $M$. racemosus, M. rouxii, M. hiemalis (minus strain), Syncephalis cornu, Zygorhynchus

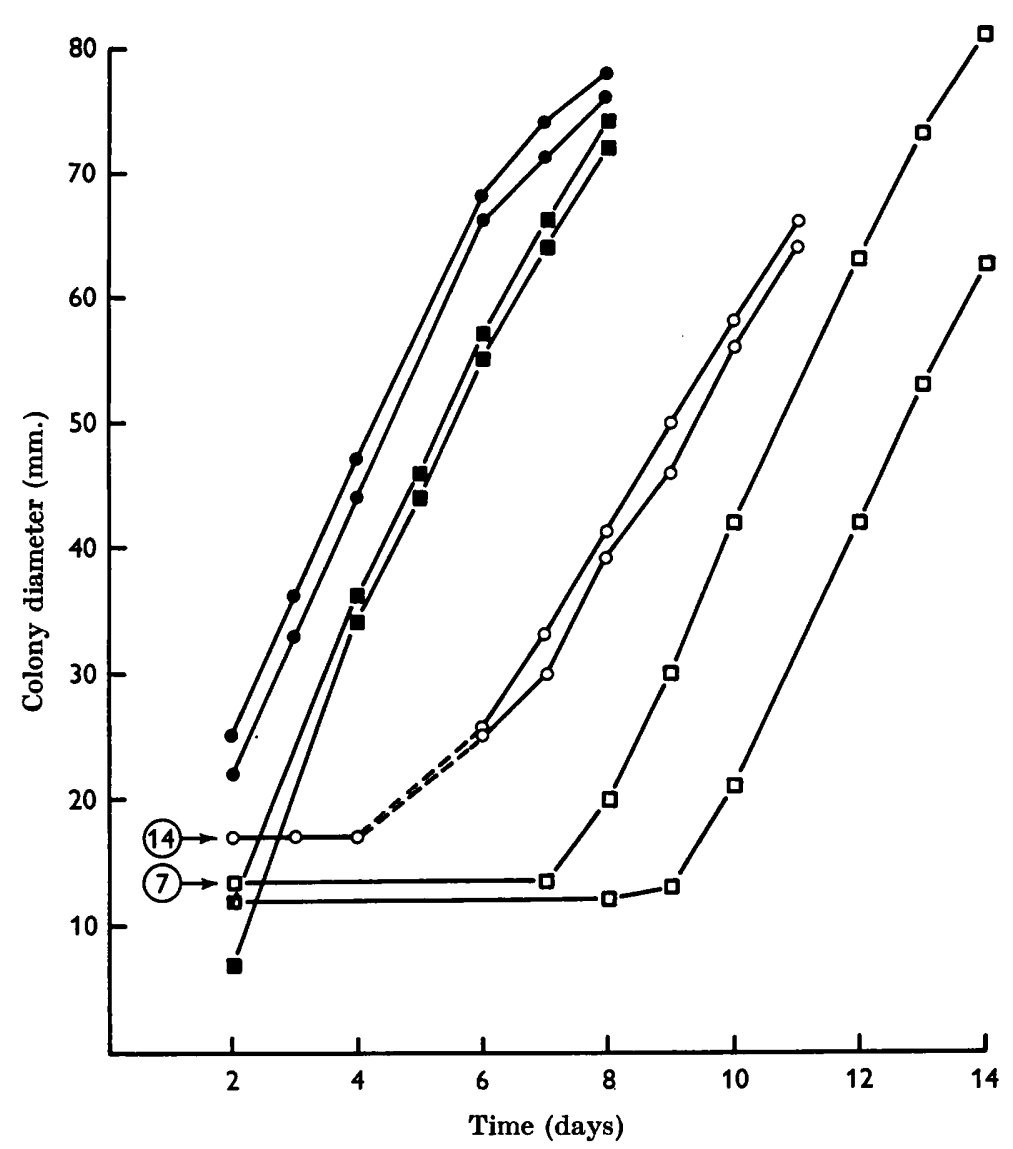

Fig. 1. Growth of Penicillium stipitatum and Circinella mucoroides after exposure to $10 \mathrm{~atm}$. of oxygen for 7 to 14 days respectively. $\square$, Penicillium stipitatum control; $\square$, $P$. stipitatum treated for 7 days; $O$, Circinella mucoroides control; $O$, C. mucoroides treated for 14 days.

exponens); 5 Ascomycetes (Chaetomium indicum, Endomycopsis capsularis, Eremascus albus, Preussia (Perisporium) funiculata, Saccharomyces cerevisiae); 1 Basidiomycete (Fomes pomaceus); and 16 Fungi Imperfecti (Ascochyta pisi, Aspergillus nidulans, $A$. ochraceus, $A$. wentii, Candida albicans, Cladosporium herbarum, Colletotrichum dematium, Penicillium camemberti, $\boldsymbol{P}$. claviforme, $\boldsymbol{P}$. janczereski, $\boldsymbol{P}$. notatum, $\boldsymbol{P}$. spinulosum, $\boldsymbol{P}$. stipitatum, $\boldsymbol{P}$. terrestre, Trichoderma viride, Verticillium lateritium).

Several points of interest arose from this preliminary survey. 
Table 1. Reaction of fungi to $10 \mathrm{~atm}$. pressure of oxygen at $25^{\circ} \mathrm{C}$

Figures indicate days after removal from pressure when cultures resumed growth. Brackets indicate that only one replicate of two recovered. Points in blank spaces indicate that replicates did not resume growth.

Classification

PHYCOMYCETES

Saprolegniales

Peronosporales

Mucorales

Entomophthorales

\section{ASCOMYCETES}

Endomycetales

Eurotiales

Erysiphales

Sphaeriales

Pezizales
Reference

E 30

IMI 82514

IMI 15406

IMI 77986

IMI 45954

IMI 21216

IMI 21217

IMI 78407

E 65

IMI 71629

IMI 35716

IMI 35044a

IMI 68072

E 104

IMI 63218

E 29

IMI 71487

E 37

IMI 40564

E 22

E 1

IMI 77606

IMI 43624

IMI 29216

E 27

NCYC 127
E 3
E 8
IMI 23657
NCYC 580
IMI 41112
E 80
E 95
E 92
E 96
E 100
E 24
E 99

E 110 IMI 61849

IMI 57472
No. of days under pressure

7 14

Phytophthora cactorum Pythium ultimum

Absidia glauca

Circinella mucoroides

Mortierella hygrophilla

Mucor hiemalis (+)

$M$. hiemalis (-)

M. hiemalis (-)

M. mucedo

M. plumbeus

M. pusillus

M. racemosus

M. ramannianus

$M$. rouxii

Phycomyces blakesleeanus

P. nitens (+)

P. nitens (-)

Rhizopus arrhizus

R. cohnii

R. oryzae

$\boldsymbol{R}$. stolonifer

Syncephalastrum cinereum

Syncephalis cornu

Thamnidium elegans

Zygorhynchus exponens

Entomophthora sp.

(8)

Endomycopsis capsularis

Eremascus albus

Saccharomyces cerevisiae

Schizosaccharomyces octosporus

Zygosaccharomyces bailii

Gymnoascus subumbrinus

(11)

Preussia (Perisporium)

funiculata

Chaetomium indicum

Glomerella cingulata

G. tucumanensis

Mycosphaerella macrospora

Sordaria fimicola

Venturia inaequalis

Diplocarpon rosae

Sclerotinia fructigena

Pyronema domesticum 
Table 1 (cont.)

Classification

BASIDIOMYCETES

Agaricales

FUNGI IMPERFECTI

Sphaeropsidales

Melanconiales

Moniliales
Reference

Fungus

Fomes pomaceus

E 20

IMI 79125

Merulius lacrymans

$\begin{array}{ll}\text { E 14 } & \text { Ascochyta pisi } \\ \text { IMI 17361 } & \text { Phoma alternariaceum } \\ \text { IMI 45944 } & \text { P. eupyrena } \\ \text { IMI 46259 } & \text { P. glomerata } \\ \text { IMI 49948 } & \text { P. violacea }(\text { P. pigmenti- } \\ & \text { vora) } \\ \text { E 91 } & \text { Phyllosticta sorghina } \\ \text { E 84 } & \text { Pyrenochaeta terrestris } \\ \text { E 86 } & \text { Selenophoma donacis }\end{array}$

Colletotrichum dematium C. gossypii

E 94

E 44

E 28

E 73

IMI 17455

E 48

E 47

E 46

E 51

E 10

E 50

E 67

E 53

E 52

E 45

E 32

E 33

IMI 89362

E 25

E 23

E 83

E 81

E 82

IMI 68412

IMI 53994

IMI 45769

IMI 53993

IMI 61868

IMI 53252

IMI 102237

E 60

IMI 27831

E 57

E 64

E 55

E 61

E 61

E 59

E 26
Alternaria brassicae

A. brassicicola

A. tenuis

Aspergillus amstelodami

A. chevalieri

A. flavus

A. fumigatus

A. nidulans

A. niger

A. ochraceus

A. quercinus

A. tamarii

A. terreus

A. wentii

Botrytis allii

$B$. cinerea

Caldariomyces fumago

Candida albicans

Cladosporium herbarum

Epicoccum purpurascens

Fusarium avenaceum

F. merismoides

F. solani

Helminthosporium

australiense

$H$. cynodontis

H. harwaiiense

H. rostratum

H. sorghicola

H. torulosum

Penicillium brevi-compactum

P. camembertii

$P$. chrysogenum

P. claviforme

P. cyclopium

$P$. cylindrosporium

$P$. cylindrosporium

$P$. decumbens

$P$. janczereskii
No. of days under pressure

$7 \quad 14$

(9)

\section{2}

\section{•}

.

(7)

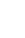


Table 1 (cont.)

\begin{tabular}{|c|c|c|c|c|}
\hline \multirow[b]{2}{*}{ Classification } & \multirow[b]{2}{*}{ Reference } & \multirow[b]{2}{*}{ Fungus } & \multicolumn{2}{|c|}{$\begin{array}{l}\text { No. of days } \\
\text { under pressure }\end{array}$} \\
\hline & & & 7 & 14 \\
\hline & IMI 15378 & P. notatum & 8 & - \\
\hline & E 54 & P. spinulosum & $\mathbf{3}$ & . \\
\hline & IMI 69250 & P. stipitatum & $5-6$ & . \\
\hline & E 56 & P. terrestre & (4) & - \\
\hline & E 58 & $P$. varioti & . & (9) \\
\hline & E 62 & $P$. velutinum & - & $5-6$ \\
\hline & E 62 & $P$. velutinum & . & $7-9$ \\
\hline & E 101 & $\begin{array}{l}\text { Aureobasidium pullulans } \\
\text { (Pullularia pullulans) }\end{array}$ & . & $\cdot$ \\
\hline & E 98 & Stilbum sp. & - & . \\
\hline & E 97 & Trichoderma koningii & . & . \\
\hline & IMI $4 \mathbf{4 5 5 3}$ & T. viride & 2 & . \\
\hline & E 38 & Trichothecium roseum & • & . \\
\hline & IMI 54377 & Verticillium lateritium & 4-5 & . \\
\hline
\end{tabular}

$\mathrm{IMI}=$ Commonwealth Mycological Institute; $\mathrm{NCYC}=$ National Collection of Yeast Cultures; $\mathbf{E}=$ Fungus collection, Department of Botany, University of Exeter.

1. Relationship between reaction of a fungus to treatment and its taxonomic position. There appears to be no correlation between the taxonomic position of a fungus and its reaction to treatment. Thus two mucor species withstood $10 \mathrm{~atm}$. of oxygen for 14 days, two withstood pressure for 7 but not 14 days, and two did not withstand 7 days (Table 1). At least one member of each of the Classes Phycomycetes, Ascomycetes and Fungi Imperfecti recovered from exposure for 14 days to 10 atm. of oxygen, and all Classes had members which recovered after 7-day treatments. A relatively large number of species of the genera Aspergillus and Penicillium were investigated, and here a high proportion (14/25) were resistant to exposure for 14 days. A smaller proportion (9/25) withstood exposure for 7 days, and only two (P. brevicompactum and $\boldsymbol{P}$. camembertii) did not recover after either treatment.

2. Growth rates of control and treated fungi. The criterion of growth rate was increase in colony diameter; measurement of two control colonies gave the normal growth rate for each fungus. While in the pressure vessel, the growth of all colonies ceased, but when treated colonies resumed growth after removal from pressure the growth rate after recovery was the same as in control colonies. Typical graphs are presented in Fig. 1 for Penicillium stipitatum after a 7-day treatment and for Circinella mucoroides after a 14-day treatment. As soon as growth was resumed, the rate was the same as if there had been no intermediate exposure to high oxygen pressure.

3. Recovery of colonies more than 14 days after treatment. For the most part, cultures were kept under observation for 14 days after treatment and then discarded. Over this period, the lag time between removal from pressure and resumption of growth varied with different fungi, and was as much as 13 days with Achlya bisexualis and Zygorhynchus exponens (Table 1). On one occasion cultures of Schizosaccharomyces octosporus, Rhizopus arrhizus, R. stolonifer, Phytophthora cactorum, Alternaria brassicicola, Botrytis allii and B. cinerea, which had been exposed to $10 \mathrm{~atm}$. of oxygen for 7 days, were retained for 32 days after treatment. Although 
none of the other cultures resumed growth, one replicate of Schizosaccharomyces octosporus recovered between 14 and 32 days after removal from pressure (estimated at 25 days by extrapolation from the growth curve). It must be stressed, therefore, that the results in Table 1 , other than that for $S$. octosporus, refer to observations up to 14 days after treatment only. Perhaps some fungi would have recovered in a longer period.

4. Variability in recovery of replicates. In Table 1 there are some instances in which only one replicate of two recovered after treatment (figures in brackets) and, in a few instances where the treatments were repeated, it was found that behaviour of the treated fungi differed at different times. As an example of the latter, the first time two replicates of the minus strain of Mucor hiemalis were exposed for 7 days to $10 \mathrm{~atm}$. of oxygen, one only resumed growth 6 days after removal from pressure; on repeating the experiment, both replicates recovered 3 days after removal from pressure. Aspergillus niger was very variable in this respect. The first result of a 14-day exposure was that neither replicate recovered; in a second experiment, both resumed growth after 3 days; in a third, neither recovered; in a fourth, both recovered in 3 days. In a larger experiment with 6 replicates, 2 recovered in 10 days, 1 in 16 days, 1 in 19 days after treatment, and 1 did not recover within 34 days of treatment. Furthermore, there was some variation between replicates even when both did resume growth after treatment. Usually they did so together, but in some cases there was a lag of a day, or at most 2 days, between recovery times (see e.g. Mucor ramannianus and Penicillium cyclopium in Table 1 ).

It appeared possible that the position of a culture in the pressure vessel might be related to its subsequent behaviour. Consequently, 10 replicates of Rhizopus arrhizus were placed in a known order in a pressure vessel, no. 1 at the top, no. 10 at the bottom. They were then subjected to $10 \mathrm{~atm}$. pressure of oxygen for 4 days. Five of the ten resumed growth after treatment, namely nos. 1, 2, 3, 7, 9 and 10. Such a random distribution of recoveries would appear to argue against position in the stack as a factor which influenced recovery. In subsequent treatments, where placement was recorded, there was no evidence that this was a significant factor.

\section{Investigations of variability of response to oxygen pressure}

The several aspects of variability of response were investigated in more detail. Ten replicates of Mucor plumbeus (both replicates had recovered previously), 12 replicates of Aspergillus wentii ( 1 of 2 replicates had recovered previously) and 10 replicates of Fusarium solani (neither replicate had recovered previously) were exposed to $10 \mathrm{~atm}$. of oxygen for 7 days. All the replicates of Mucor plumbeus resumed growth 2-3 days after removal from pressure. Only 10 of the 12 replicates of $A$. wentii did so; the remaining two had not recovered after 40 days. All the replicates of $\boldsymbol{F}$. solani resumed growth 4-5 days after removal from pressure. In each experiment, all replicates had been subcultured from the same inoculum of the appropriate fungus on to the same medium, and had been treated identically.

Since the sources of the inocula were not of known age, an examination was made on the reaction of subculture originating from young and from old cultures of Mucor racemosus. Three-day and 42-day colonies were used as sources of inocula, having grown for these periods on malt agar at $25^{\circ}$. Five subcultures were made from each, grown for 2 days, then placed under oxygen at 10 atm. for 7 days. All 


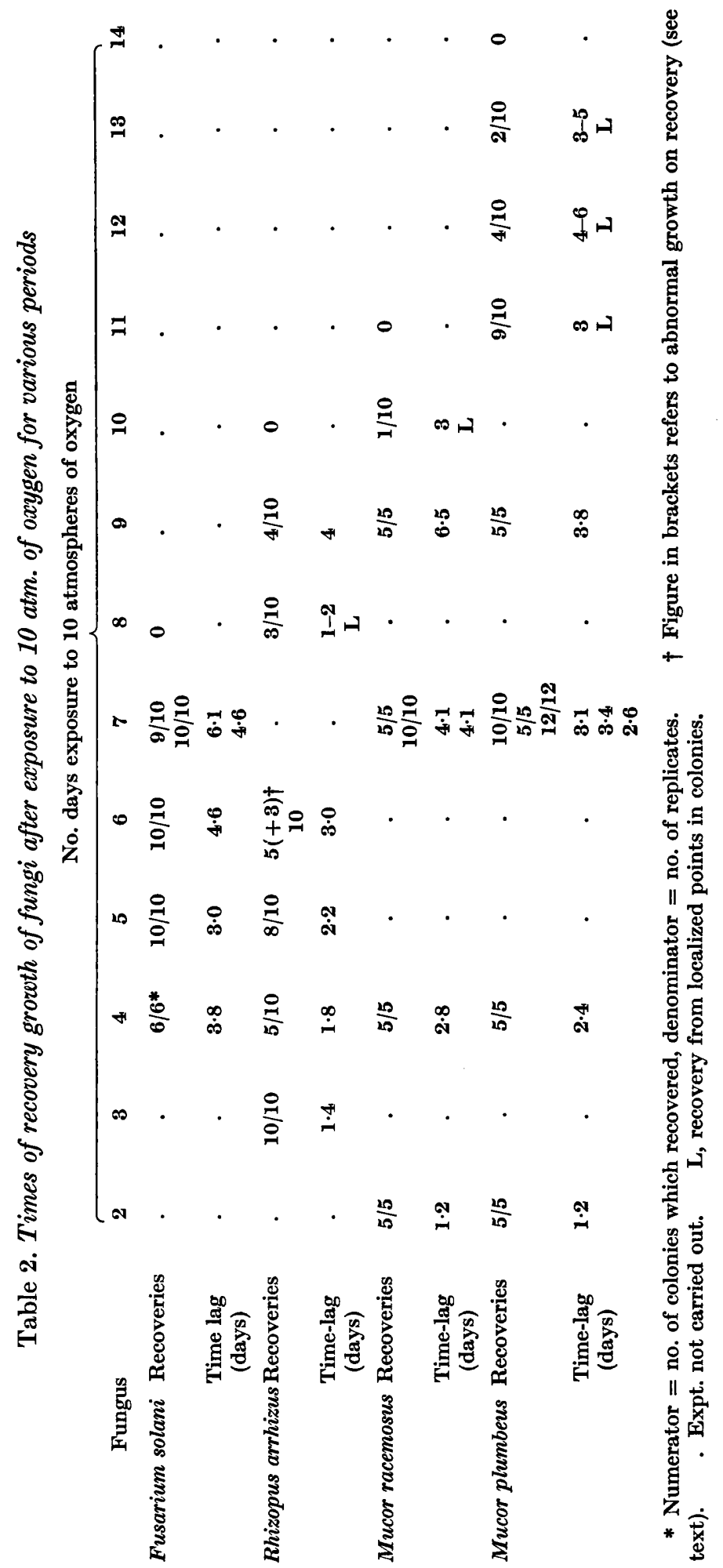


the replicates recovered, but those from the young inocula did so in an average of $5 \cdot 4 \pm 0 \cdot 6$ days while those from the old inocula took $4 \cdot 4 \pm 0.5$ days. This result is significant at the $5 \%$ level. In a similar experiment with 5 replicates each of inocula from a 7-day and from a 171-day culture of $M$. plumbeus, all resumed growth at the same time 4 days after removal from pressure. It would appear therefore that although inocula taken from old colonies of $M$. racemosus were slightly more resistant to high oxygen pressure than those of young colonies, there was no significant difference in this respect with $M$. plumbeus.

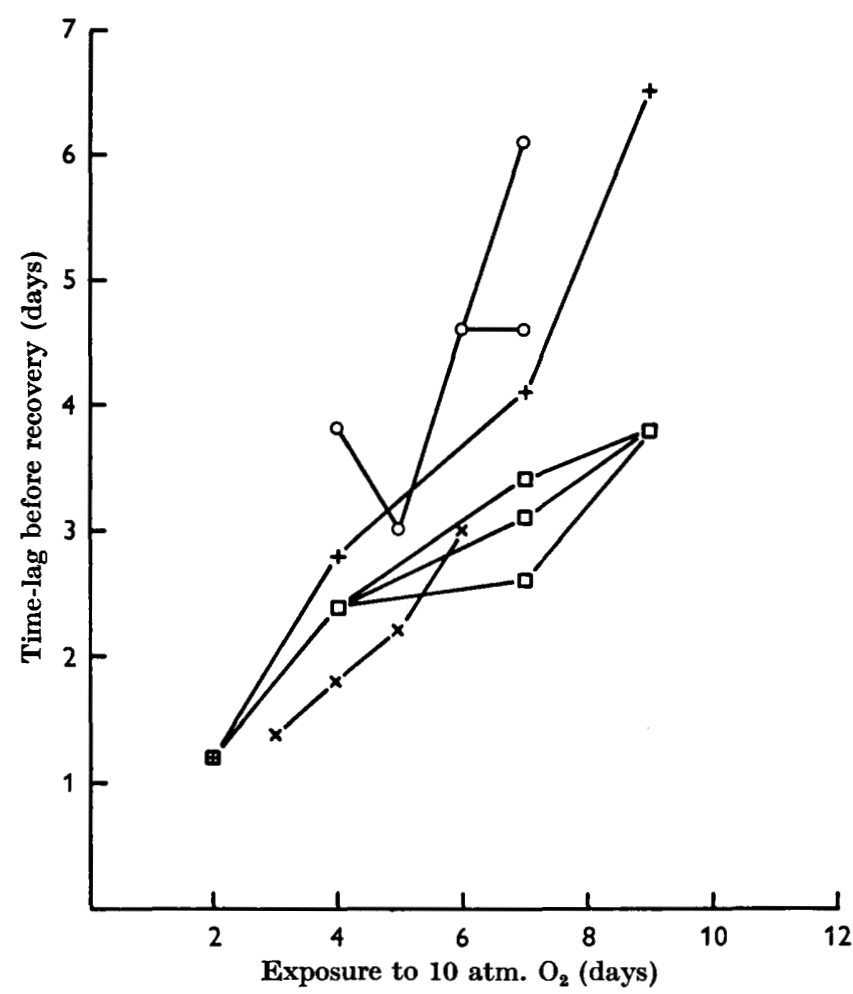

Fig. 2. Relationship between length of exposure to $10 \mathrm{~atm}$. oxygen and time-lag before recovery. O, Fusarium solani; $\times$, Rhizopus arrhizus; +, Mucor racemosus; $\square$, Mucor plumbeus.

More detailed investigations were made on Mucor racemosus, M. plumbeus, Rhizopus arrhizus and Fusarium solani, with a procedure modified in the light of previous experience. The modifications were: $(a)$ to use at least 5 replicates of each fungus; $(b)$ to extend observations over at least 21 days after treatment in cases of non-recovery; $(c)$ to standardize the inocula by always taking them from 3-day colonies. The object of these experiments was to relate time of exposure to oxygen under pressure to the lag time before recovery, and to find the extinction point at which the fungus did not recover from the oxygen pressure treatment. The results of these experiments are summarized in Table 2.

It is clear from Table 2 that longer exposures to oxygen at $10 \mathrm{~atm}$. pressure were in general correlated with longer lag periods before growth under normal conditions was resumed. This is more clearly shown in Fig. 2. 
The extinction point (i.e. the number of days of exposure to oxygen at $10 \mathrm{~atm}$. pressure which killed all replicates) was 8 days for Fusarium solani, 10 days for Rhizopus arrhizus, 11 days for Mucor racemosus and 14 days for $M$. plumbeus. With $F$. solani this was very clear-cut. All replicates recovered from exposure to $\mathrm{O}_{2}$ pressure for 7 days whereas none recovered from exposure for 8 days. On repeating the experiments, 9 of 10 replicates recovered from the 7-day treatment while none recovered from the 8-day treatment. The behaviour of the other three fungi was not so clear-cut. All replicates of $M$. racemosus recovered from a 9-day treatment, 1 of 10 from a 10-day treatment, and none from an 11-day treatment. The one culture which recovered from the 10-day treatment appeared to resume growth at one localized spot within the colony; from this spot, which was located in the sporing centre of the original colony, a new colony developed which spread over the original, apparently dead mycelium. It looked very much as if a spore or a group of spores had survived the treatment and had later resumed growth.

There was some evidence of a similar selective process in colonies of Mucor plumbeus after exposure to $10 \mathrm{~atm}$. of oxygen for 11, 12 and 13 days. This treatment was very near the extinction point for this fungus, which is 14 days. After the 11-day treatment, it was observed on the third day after removal from pressure that centres of growth appeared in the sporing region of some colonies; only 1 colony did not recover at all, and the remaining 9 replicates resumed growth at 1-6 or more distinct loci. After the 12-day treatment, 4 out of 10 replicates showed a similar pattern of growth, but here growth was resumed 4-6 days after removal from pressure; in 2 colonies there was one locus, and in the other two there were 2 loci. After the 13-day treatment, 2 of 10 replicates resumed growth, each at one locus, 3-5 days after treatment. This new growth spread over the non-growing mycelial rim of the original colony, and where there was more than one centre of growth, remained distinctly lobed for several days. Eleven 'strains' were isolated from 5 plates which had received treatment for 11 days, and two replicates of each 'strain' subjected to treatment for 12 days. In two instances both replicates resumed growth 3-4 days after treatment at a single locus in the sporing centre of each colony; the colony as a whole did not resume growth.

Rhizopus arrhizus showed very variable behaviour. Five replicates of 10 recovered from a 4-day exposure and 8 of 10 from a 5-day exposure. After a 6-day exposure, 5 of 10 replicates recovered normally (i.e. apparently the whole colony resumed growth at the same time) 3 days after removal from pressure. Two of the remaining 5 replicates did not resume growth within 35 days after $\mathrm{O}_{2}$ pressure treatment. However, localized centres of recovery appeared in the other replicates 9-14 days after removal from pressure, but the new growth was atypical; instead of being rapid and producing a fluffy aerial mycelium it developed as a slow-growing, semi-submerged or superficial mycelium. On examination, the hyphae were found to be disorganized internally, and to have a stunted growth form. After developing for a few days in this manner, all growth ceased in the plates, although subcultures from these colonies grew in tubes in a similar manner for a few days before also ceasing to develop. Of 10 replicates from the normal stock colony of $\boldsymbol{R}$. arrhizus subjected to pressure for 8 days, 3 resumed growth from localized recovery points 2 days after exposure. Here growth was normal and rapid. After a 9-day exposure, 4 of 10 replicates resumed growth 5 days after treatment. After a 10-day exposure there was no recovery. 
The presence of localized recovery points observed in replicates of Mucor racemosus, $M$. plumbeus and Rhizopus arrhizus, all of which were sporing at the time of exposure to oxygen pressure, seems to indicate that growth was resumed by resistant cells or spores. If this were so, colonies arising from such selected hyphae or spores would be expected to give subcultures which were also resistant. This was investigated by using $R$. arrhizus as the test organism. Five replicates subcultured from a 3-day stock culture were grown for one day. Two further subcultures were then taken from each replicate before it was subjected to oxygen pressure for 6 days. These secondary subcultures (A 1, A 2, B 1, B 2, etc.) after growing for one day, were then subjected to the same treatment. After removal from pressure, three of the original cultures, (A, B, C) and one of the subcultures (B 2) resumed growth. The fact that only 1 subculture resumed growth of 6 from the original cultures A, B and $\mathbf{C}$ does not strongly support the idea that ability to recover is a 'strain' characteristic. However, since each of the cultures A-E may have consisted of a mixture of strains, the result cannot be taken as disproving the existence of strains with greater powers of recovery than others. If such strains do exist, it is more likely that they would be detected by taking subcultures only from cultures which recovered after treatment. Therefore a further set of subcultures (A 3-6, B 3-6, C 3-6) from colonies $\mathrm{A}, \mathrm{B}$ and $\mathrm{C}$ which had recovered after treatment, were grown for one day, then subjected to the same treatment. After removal from oxygen pressure, 3 of these subcultures (C 3, C 5, C 6) resumed growth. Although this result is also inconclusive, the fact that 3 of 4 subcultures from one source did recover suggests that the treatment may have some selective effect on the colonies.

\section{DISCUSSION}

Of 103 fungi tested in the present study, 52 recovered from exposure to $10 \mathrm{~atm}$. oxygen for 7 days, and of these, 22 withstood exposure for 14 days. Resistant species were not confined to any one Class nor to a limited number of genera. It would appear therefore that fungi are substantially more resistant to high oxygen pressures than are plant (Caldwell, 1956, 1964) and animal (Bean, 1945) tissues, although not so resistant as some bacteria (Caldwell, 1965).

When the fungal colonies resumed growth after treatment, it was found that the subsequent growth rates, measured as increase in colony diameter, were the same as that of control colonies not subjected to high oxygen pressure. Since there was a lag period between treatment and resumption of growth, it would appear that the suspension of certain metabolic systems, presumably by oxidation induced by high oxygen pressure, requires a period of adjustment after removal from pressure before the complete metabolic cycle and hence growth can be resumed. One such system, which appears to be of variable sensitivity in different fungi, has been found by Dr G. G. Pritchard of this Department to be the pyruvate oxidase system (private communication). The period of adjustment may be a lengthy one (21-28 days in Schizosaccharomyces octosporus after a 7-day treatment), the time generally being correlated with the time of exposure to high oxygen pressure, longer exposures resulting in longer lag periods. This agrees with Caldwell's observations on fungal recovery (Caldwell, 1963).

Caldwell (1965), working with bacteria, noted that after 28-day exposure it was 
possible that not all the bacteria, but only some individuals of the colony, perhaps strains, survived. McAllister et al. (1963) refer to 'hyperbars', which may be individuals selected or selectively altered by the treatment. Such selective survival was noted in the present work, with the three fungi Rhizopus arrhizus, Mucor racemosus and $M$. plumbeus, when exposure times approached the extinction point, which was 10 days, 11 days and 14 days, respectively; and selective survival was first apparent after exposures of 8 days, 10 days and 11 days, respectively. Exposure of $R$. arrhizus for 6 days did result in a degree of selectivity in that 3 cultures out of 10 replicates resumed growth 9-14 days after treatment. The new growth, however, was then very abnormal, with internally disorganized hyphae; this is regarded as selective damage rather than selective survival.

At first it appeared likely that selection occurred from spores or from strains in a mixture either by chance from the stock colony during subculture, or as a result of exposure of replicates to high oxygen pressure. Both possibilities were examined with Rhizopus arrhizus, but with inconclusive results. Nevertheless, variability of response of replicates to high oxygen pressure is puzzling. When only two replicates were used, as was the case for most of the fungi investigated here, there were several occasions when only one resumed growth after treatment, or, when both recovered, there was a difference in the lag period of 1 or 2 days. Furthermore, in the initial survey (Table 1 ) both replicates of $R$. arrhizus and of Fusarium solani did not recover from a 7-day treatment, whereas in later experiments with a larger number of replicates the extinction points were 10 days and 8 days, respectively. Aspergillus niger was perhaps the most variable fungus, the replicates either not resuming growth at all or recovering at times varying from 3 to 19 days after a 14-day exposure to high oxygen pressure. This suggests that some variability also existed in the initial subcultures.

Fairly consistent results were obtained with Fusarium solani, Mucor racemosus and $M$. plumbeus (Table 2) using the modified procedure described on p. 25, except when exposure time approached their extinction points. Rhizopus arrhizus on the other hand was much less reliable even with the modified procedure. It is likely that there are many strains in the stock cultures of $\boldsymbol{R}$. arrhizus and that this causes the wide variation of response to treatment.

The author is indebted to Professor J. Caldwell, Dr G. G. Pritchard and DrS. A. J. Tarr for helpful advice and criticism during the course of this work.

\section{REFERENCES}

Bean, J. W. (1945). Effects of oxygen at increased pressure. Physiol. Rev. 25, 1.

Bert, P. (1878). La Pression Barometrique. Paris: G. Masson.

Boerema, I., Brummelkamp, W. H. \& Muejne, N. G. (1964). Proc 1st int. Congr. on Clinical application of Hyperbaric Oxygen, Sept. 1963. Amsterdam.

Caldwell, J. (1956). Studies in the respiration of apples at various pressures of oxygen. J. exp. Bot. 7, 326.

Caldwell, J. (1963). Effects of high partial pressures of oxygen on fungi. Nature, Lond. 197, 772.

Caldwell, J. (1964). Effects of high pressure of pure oxygen on tissues. Nature, Lond. 201, 514.

Caldweld, J. (1965). Effects of high partial pressures of oxygen on fungi and bacteria. Nature, Lond. 206, 321. 
Camprell, J. A. (1937). Body temperature and oxygen poisoning. J. Physiol. 89, $17 P$.

Cleveland, L. R. (1925). Toxicity of oxygen for protozoa in vivo and in vitro: animals defaunated without injury. Biol. Bull. 48, 455.

Faulkner, J. M. \& Binger, C. A. L. (1927). Oxygen poisoning in cold-blooded animals. J. exp. Med. 45, 865.

Hutchison, J. H., Kerr, M. M., Williams, K. G. \& Hopkins, W. I. (1963). Hyperbaric oxygen in the resuscitation of the new-born. Lancet ii, 1019.

McAllister, T. A., Stark, J. M., Norman, J. N. \& Ross, R. M. (1963). Inhibitory effects of hyperbaric oxygen on bacteria and fungi. Lancet ii, 1040.

Thaysen, A. C. (1934). Preliminary note on the action of gases under pressure on the growth of microorganisms. I. Action of oxygen under pressure at various temperatures. Biochem. J. 28, 1330. 